\title{
Improving plant stress-tolerance: potential applications of engineered MAPK cascades
}

\author{
Khaled Moustafa*
}

\begin{abstract}
There will be a paramount need to develop solutions to the problem of worldwide food crop scarcity under exacerbated climate change. This motivates an effort to leverage agricultural biotechnology to improve plant adaptation to severe and multiple, simultaneous environmental stresses. Consequently, engineering synthetic signaling pathways, particularly mitogen activated protein kinase (MAPK) cascades utilizing components already involved in plant stress tolerance, is a worthy focus for research to breed new plant varieties with enhanced stress-tolerance traits.
\end{abstract}

Keyword: MAP kinase, mitogen activated protein kinase, MAPK transgenic circuit, environmental stress, plant adaptation.

\section{Rationale for engineering plant MAPKs to enhance stress tolerance}

MAPKs are involved in multiple environmental biotic and abiotic stress signaling pathways, reviewed in (Pitzschke et al., 2009) (Rodriguez et al., 2010) (Moustafa et al., 2014). Overexpression of single MAPKs genes in plants yields positive effects on stress adaptation in vitro and under laboratory conditions, but results of single genes approach under field conditions are still below expectations (Castiglioni et al., 2008, Mittler, 2006). This suggests that a single gene approach is not sufficient to produce inherently stressadapted varieties. Overexpression of a single MAPK gene may result in a mismatch in stoichiometry between the gene product and its target, leading to inefficient signal transduction and poor cellular function. Overexpression of all components of a MAPK cascade (receptor, adaptors, kinases, effectors), would ensure correct stoichiometry. Augmenting the concentrations of MAPK components can also increase the sensitivity and decrease the activation threshold of a cascade (O'Shaughnessy et al., 2011). Additionally, given the similarity and conservation of components from different MAPK pathways (Widmann et al., 1999), components from one pathway or plant species could be used in another, or multiple pathways could be connected. These "rewired" components could comprise one or more synthetic MAPK circuits to generate new plant genotypes with improved adaptability to multiple environmental stresses. The output of such circuits can be assessed by measuring the amount of osmolytes and reactive oxygen species under both biotic and abiotic stress conditions. 


\section{How would a synthetic MAPK cascade improve plant stress tolerance?}

Gene candidates for engineered MAPK cascades

The ABA receptor (Kim et al., 2014) (Okamoto et al., 2013), the kinases MKKK20 (Kim et al., 2012), ZmMKK1/ZmMKK3 (Cai et al., 2014) (Zhang et al., 2012), and ZmMPK5/ZmSIMK1 (Zhang et al., 2014) (Wang et al., 2014), and the transcription factors WRKY (Okay et al., 2014, Pan and Jiang, 2014) were all implicated in plant responses to environmental stress. This provides a diverse repertoire of candidate genes for assembling into synthetic circuits (Figure 1) and testing for improved plant stress tolerance. To improve plant resistance to biotic stresses, a complete MAPK cascade comprising the flagellin receptor (FLS2), the kinases MEKK1, MKK4/MKK5, and MPK3/MPK6, and the transcription factor WRKY (Asai et al., 2002) would be a worthy choice for engineering. In an abiotic stress context, a good candidate is an incomplete cascade comprising the kinases MEKK1, MKK2, and MPK4/MPK6) (Teige et al., 2004). Although this cascade lacks a receptor and effector, the core components could still be used.

\section{Potential crosstalk with native pathways}

A major concern of a synthetic MAPK circuit is the potential crosstalk between the synthetic circuit and the native signal pathways, which may produce unexpected or deleterious effects. One possible strategy to circumvent any potential undesirable crosstalk is to design synthetic circuits as orthogonal or heterologous modules, utilizing components from other organisms, which would not interact with native signaling components. However, many multiplex transgenic corn lines were created, expressing at least five genes involved in vitamin metabolic pathways, with no undesirable effects observed (Naqvi et al., 2009). An entire metabolic pathway was engineered in Arabidopsis with only minor inadvertent effects on the metabolome and transcriptome (Kristensen et al., 2005). In bacteria, the rewiring of promoters with different transcription or sigma factor genes in about 600 combinations was also tolerated by the organism with only minor effects on growth (Isalan et al., 2008).

\section{Alternative deployment of synthetic MAPK circuits}

In addition to responding to natural stimuli, synthetic MAPK circuits can be engineered to respond to non-natural stimuli by linking MAPK component gene expression to inducible promoters, driven by molecules such as estradiol (O'Shaughnessy et al., 2011). Estradiolinduced gene expression of the heat-shock factor HSFA4A confers enhanced tolerance to salt and oxidative stresses in Arabidopsis (Pérez-Salamó et al., 2014). This demonstrates the viability of exogenous applications of appropriate inducers to drive gene expression in whole plants. In the field, the circuits could be activated by spraying plants with a solution containing the appropriate inducers (e.g. ligand, abscissic acid or others) at the concetrations determined to give the best output, similar to how we currently deliver some pesticides or herbicides. Another option is to dissolve the inducers in the crop irrigation water. 


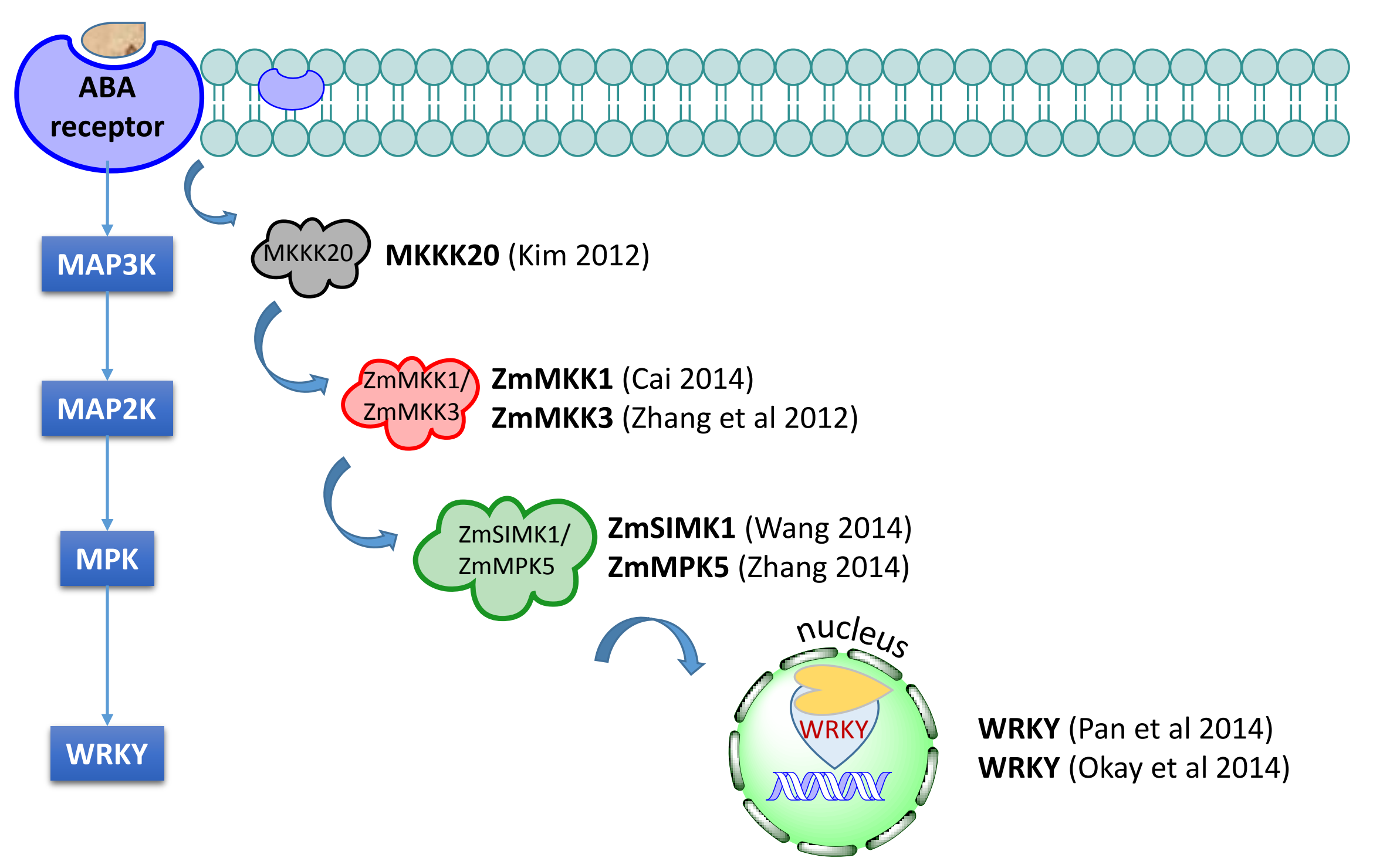

Figure 1. A synthetic MAPK transgenic circuit could be engineered from genes involved in stress responses, from receptor to targeted transcription factor. ABA receptor (Kim et al., 2014) (Okamoto et al., 2013), MKKK20, ZmMKK1/3, ZmSIMK1/ZmPK5 and WRKY transcription factors are all involved in environmental stress-responses; they could be assembled from different plant species into a synthetic MAPK transgenic circuit (or synthetic signaling modules) to transform plant and assess plantstress tolerance : 


\section{References}

1. Asai, T., Tena, G., Plotnikova, J., Willmann, M.R., Chiu, W.L., Gomez-Gomez, L., . . Sheen, J. (2002) MAP kinase signalling cascade in Arabidopsis innate immunity. Nature 415, 977-983.

2. Cai, G., Wang, G., Wang, L., Pan, J., Liu, Y., and Li, D. (2014) ZmMKK1, a novel group A mitogen-activated protein kinase kinase gene in maize, conferred chilling stress tolerance and was involved in pathogen defense in transgenic tobacco. Plant science : an international journal of experimental plant biology 214, 57-73. DOI: 10.1016/j.plantsci.2013.09.014.

3. Castiglioni, P., Warner, D., Bensen, R.J., Anstrom, D.C., Harrison, J., Stoecker, M., . . Heard, J.E. (2008) Bacterial RNA chaperones confer abiotic stress tolerance in plants and improved grain yield in maize under water-limited conditions. Plant physiology 147, 446-455.

4. Isalan, M., Lemerle, C., Michalodimitrakis, K., Horn, C., Beltrao, P., Raineri, E., . . Serrano, L. (2008) Evolvability and hierarchy in rewired bacterial gene networks. Nature 452, 840-845.

5. Kim, H., Lee, K., Hwang, H., Bhatnagar, N., Kim, D.Y., Yoon, I.S., . . . Kim, B.G. (2014) Overexpression of PYL5 in rice enhances drought tolerance, inhibits growth, and modulates gene expression. Journal of experimental botany 65, 453-464. DOI: 10.1093/jxb/ert397

6. Kim, J.-M., Woo, D.-H., Kim, S.-H., Lee, S.-Y., Park, H.-Y., Seok, H.-Y., . . Moon, Y.-H. (2012) Arabidopsis MKKK20 is involved in osmotic stress response via regulation of MPK6 activity. Plant cell reports 31, 217-224.

7. Kristensen, C., Morant, M., Olsen, C.E., Ekstrom, C.T., Galbraith, D.W., Moller, B.L., and Bak, S. (2005) Metabolic engineering of dhurrin in transgenic Arabidopsis plants with marginal inadvertent effects on the metabolome and transcriptome. Proceedings of the National Academy of Sciences of the United States of America 102, 1779-1784.

8. Mittler, R. (2006) Abiotic stress, the field environment and stress combination. Trends in plant science 11, 15-19.

9. Moustafa, K., AbuQamar, S., Jarrar, M., Al-Rajab, A.J., and Tremouillaux-Guiller, J. (2014) MAPK cascades and major abiotic stresses. Plant cell reports. DOI: 10.1007/s00299-0141629-0

10. Naqvi, S., Zhu, C., Farre, G., Ramessar, K., Bassie, L., Breitenbach, J., . . Christou, P. (2009) Transgenic multivitamin corn through biofortification of endosperm with three vitamins representing three distinct metabolic pathways. Proceedings of the National Academy of Sciences of the United States of America 106, 7762-7767.

11. O'Shaughnessy, E.C., Palani, S., Collins, J.J., and Sarkar, C.A. (2011) Tunable Signal Processing in Synthetic MAP Kinase Cascades. Cell 144, 119-131.

12. Okamoto, M., Peterson, F.C., Defries, A., Park, S.Y., Endo, A., Nambara, E., . . . Cutler, S.R. (2013) Activation of dimeric ABA receptors elicits guard cell closure, ABA-regulated gene expression, and drought tolerance. Proceedings of the National Academy of Sciences of the United States of America 110, 12132-12137.

13. Okay, S., Derelli, E., and Unver, T. (2014) Transcriptome-wide identification of bread wheat WRKY transcription factors in response to drought stress. Molecular genetics and genomics : MGG. DOI: 10.1007/s00438-014-0849-x

14. Pan, L.J. and Jiang, L. (2014) Identification and expression of the WRKY transcription factors of Carica papaya in response to abiotic and biotic stresses. Mol Biol Rep 41, 1215-1225. DOI: 10.1007/s11033-013-2966-8

15. Pérez-Salamó, I., Papdi, C., Gábor, R., Zsigmond, L., Vilela, B., Lumbreras, V., . . Szabados, L. (2014) The Heat Shock Factor HSFA4A confers salt tolerance and is regulated by oxidative stress and the MAP kinases, MPK3 and MPK6. Plant physiology. DOI: 10.1104/pp.114.237891

16. Pitzschke, A., Schikora, A., and Hirt, H. (2009) MAPK cascade signalling networks in plant defence. Current opinion in plant biology 12, 421-426.

17. Rodriguez, M.C., Petersen, M., and Mundy, J. (2010) Mitogen-activated protein kinase signaling in plants. Annu Rev Plant Biol 61, 621-649. 
18. Teige, M., Scheikl, E., Eulgem, T., Doczi, R., Ichimura, K., Shinozaki, K., . . Hirt, H. (2004) The MKK2 pathway mediates cold and salt stress signaling in Arabidopsis. Mol Cell 15, 141-152.

19. Wang, L., Liu, Y., Cai, G., Jiang, S., Pan, J., and Li, D. (2014) Ectopic expression of ZmSIMK1 leads to improved drought tolerance and activation of systematic acquired resistance in transgenic tobacco. J Biotechnol 172, 18-29. DOI: 10.1016/j.jbiotec.2013.11.006.

20. Widmann, C., Gibson, S., Jarpe, M.B., and Johnson, G.L. (1999) Mitogen-activated protein kinase: conservation of a three-kinase module from yeast to human. Physiological reviews 79, 143-180.

21. Zhang, D., Jiang, S., Pan, J., Kong, X., Zhou, Y., Liu, Y., and Li, D. (2014) The overexpression of a maize mitogen-activated protein kinase gene (ZmMPK5) confers salt stress tolerance and induces defence responses in tobacco. Plant biology 16, 558-570.

22. Zhang, M., Pan, J., Kong, X., Zhou, Y., Liu, Y., Sun, L., and Li, D. (2012) ZmMKK3, a novel maize group $B$ mitogen-activated protein kinase kinase gene, mediates osmotic stress and ABA signal responses. J Plant Physiol 169, 1501-1510.

\footnotetext{
*Email: khaled.moustafa@gmail.com
} 